\title{
Measuring Change in Activity Participation of Mental Health Care Users Attending an Occupational Therapy Programme in Rural South Africa
}

\author{
*Olindah Silaule. BSc Occ Ther (Wits), MSc Occ Ther (Wits). https://orcid.org/0000-0003-0406-9788 \\ Lecturer, Department of Occupational Therapy, School of Therapeutic Sciences, University of the Witwatersrand, Johannesburg, South Africa.

\section{Daleen Casteleijn. B Occ Ther (UP), B Occ Ther (Hons) (Medunsa), PG Dip Vocational Rehabilitation (UP), Dip Higher Education and Training Practices (UP), M Occ Ther (UP), PhD (UP); https://orcid.org/0000-0002-06 I I-8662}

Associate Professor, Department of Occupational Therapy School of Therapeutic Sciences, University of the Witwatersrand, Johannesburg, South Africa.

Introduction: Lack of evidence on the effect of interventions is one of the several ongoing challenges facing healthcare services in South Africa. The increasing emphasis on routine outcome measurement has urged occupational therapists to demonstrate the impact of their intervention in mental health. This study aimed to evaluate the change in activity participation of the mental health care users attending an occupational therapy programme.

Methods: A one-group pre/post-test design was used. The Activity Participation Outcome Measure (APOM) was administered to measure activity participation of 64 mental health care users. Five measurements were done between admission and follow-up after discharge. Results: Majority of the participants were between the ages of $20-29$ and mainly diagnosed with substance use disorders. The effect sizes, Cohen's $d(>0.80)$ and Cohen's $r(>0.37)$ demonstrated a significant positive change $(p=0.00)$ across all APOM domains during hospitalisation. An insignificant change $(p>0.05)$ was noted in the effect sizes during the follow-up stages with a substantial decline in some of the APOM domains indicating that patients were not well-integrated into their communities.

Conclusion: This study revealed the importance of measuring change in participation for shaping clinical practice, to ensure meaningful intervention, and successful integration of MHCUs in their communities.

Keywords: routine outcome measurement, activity participation, occupational therapy, mental health, rural context.

\section{INTRODUCTION}

Mental disorders are a common and leading cause of disability worldwide' ${ }^{\prime}$. According to World Health Organization (WHO) mental disorders contribute approximately $13 \%$ to the overall global burden of disease worldwide and accounts for $32.42 \%$ of years lived with disability ${ }^{2}$. In South Africa, mental disorders are counted as the third contributor to the overall disease burden ${ }^{3}$. According to Herman et al., the African National Congress (ANC ${ }^{4}$ one in six South Africans will have a mental disorder in a I2-month period. Additionally, one in three will have a mental disorder at some point in their lifetime ${ }^{4}$. The Global Burden of Disease conducted in 2016 revealed that mental disorders and substance-use disorders account for $35 \%$ of the Years Lost due to Disability in South Africa ${ }^{2}$.

People with mental disorders often exhibit long-term symptoms that influence their ability to function and contribute within their communities. Mental disorders result in impairments that often influence the ability to perform activities that the individual wishes to perform in order to pursue their life goals and fulfil their social roles ${ }^{5}$. Those afflicted by mental disorders often struggle to participate in daily life activities that are meaningful, consequently, reducing their ability to lead productive daily routines ${ }^{6}$. Limited participation in activities is associated with loss of identity, social status and close relationships ${ }^{7}$. Additionally, there is loss of opportunities for work and hope to be a contributing citizen, which is then accompanied by the worsening of symptoms ${ }^{7,8}$.
Participation in activities that are meaningful is a substantial component in the recovery of persons with mental disorders 9.10 . Occupational therapists target activity participation in the population they serve. They have crucial skills and knowledge to offer effective and efficient interventions relevant in the prevention and management of mental disorders. Evidence-based interventions demonstrate that occupational therapists can enable mental healthcare users to engage in meaningful occupations, participate in community living, and contribute to society" '. While there is acknowledgement of the unique contribution by occupational therapist in facilitating activity participation to enable recovery amongst persons with mental disorders, limited evidence exists on the effect of the services they offer particularly in rural areas where general access to health is limited. Over the years occupational therapists have had an abundance of valuable clinical contributions, regarding facilitating activity participation however; there is limited evidence to demonstrate change in clients' activity participation ${ }^{12}$.

\section{LITERATURE REVIEW}

The concept of activity participation in occupational therapy

Occupational therapy is grounded in the philosophy that humans are occupational beings with occupation as central to human sur- 
vival $^{13}$. Within mental health, occupational therapy aims to enable individuals to attain positive mental health, prevent mental illness and facilitate recovery by using meaningful occupations to promote activity participation" 6 . Activity participation is described as involvement in a life situation. This process occurs when clients are actively involved in carrying out daily activities they find purposeful and meaningful"14:629. Du Toit, cited in Van der Reyden et al, defined activity participation as: "Action on the world, originating in one's self, in one's relation to one self and the external world, the doing" $15: 276$. In their description of the International Classification of Functioning, Disability, and Health (ICF) the $\mathrm{WHO}^{16}$ describes activity and participation as two different constructs ${ }^{17}$. Activity is described as the actual doing of a task and participation is described as an involvement in life situations. These two concepts are central to human development and lived experience. When the individual encounters problems in these two constructs, it is identified as activity limitation and participation restriction ${ }^{16}$. Law ${ }^{18}$, similar to the American Association of Occupational Therapy ${ }^{14}$ and du Toit as cited by van der Reyden et al., ${ }^{15}$ combined these two constructs and highlighted that it is through the process of activity participation that individuals obtain skills and competencies, find purpose and meaning in life, as well as connect with others and communities.

The link between activity participation and health is clearly established and acknowledged as a core value of client-centred occupational therapy ${ }^{14}$. Occupational therapy interventions aim to promote health and well-being by facilitating participation in the categories of occupations including activities of daily living (ADLs), instrumental activities of daily living (iADLs), work, education, social participation, leisure, and rest and sleep ${ }^{14}$. In a study by Bryant et al., ${ }^{19}$ participants highlighted that participation in activities as part of the occupational therapy inpatient program had substantial impact on alleviating their symptoms as this distracted them from focusing on their distressing thoughts ${ }^{19}$. Similarly, a study by Foruzandeh and Parvin, revealed that participating in occupational therapy activities was associated with positive outcomes on the symptoms of those diagnosed with Schizophrenia ${ }^{20}$. Based on available evidence there is no doubt that occupational therapists have valuable contribution in facilitating activity participation and the substantial impact this has on the recovery of mental health care users (MHCUs). However, there remain a paucity of evidence to indicate the measurement of activity participation to demonstrate the effect of occupational therapy interventions in mental health ${ }^{21,22}$.

Measurement of activity participation in mental health

The overall purpose of mental health services in South Africa is to improve capacity of MHCUs to participate in roles of their choice in their community ${ }^{23}$. To achieve this goal, all mental healthcare professionals including occupational therapists should monitor and evaluate their efforts. The plan to implement National Health Insurance $(\mathrm{NHI})$ has placed emphasis on the need for the effectiveness of interventions to ensure good quality services and achievement of positive health outcomes ${ }^{3}$. To ensure good quality services, the National Mental Health Policy Framework and Strategic Plan (NMHF) calls for the planning and provision of evidence-based interventions in mental health ${ }^{23}$. In rural South Africa, a dire state of mental health services is reported where MHCUs are confronted by unsupportive and desert-like mental health services failing to meet their needs ${ }^{22,24}$. The escalating prevalence of mental disorders along with the reported poor quality of treatment calls for measurements of outcomes to ensure effectiveness of services in mental health ${ }^{25,26}$.

Occupational therapists have a responsibility to demonstrate effectiveness of their services to the clients, funders of services and the society at large ${ }^{12,27}$. To demonstrate their distinct value in mental health, occupational therapists should produce evidence for the use of meaningful activities to promote participation in everyday life ${ }^{6}$. Measuring the effectiveness of interventions is important as it enables occupational therapists to provide sound evidence for the services they offer. Implementation of routine outcome measurement (ROM) into routine clinical practice is fundamental to demonstrating the effectiveness of interventions ${ }^{26}$. ROM has been identified as a critical component in the facilitation of recovery in mental disorders where progress is not always identifiable ${ }^{28}$. Carlier and Eeden ${ }^{29}$ describe ROM as a repeated assessment of a service user's health status over the course of treatment or an intervention. For occupational therapists in mental health, this entails administering an outcome measurement tool on the MHCUs before, during and after an intervention in order to monitor the change in activity participation ${ }^{26}$.

A number of outcome measures are available and have been widely researched and reported in the occupational therapy literature. These outcome measures includes the Model of Human Occupation Screening Tool (MOHOST) ${ }^{30}$; Assessment of Motor and Process Skills (AMPS) ${ }^{31}$; Canadian Occupational Performance Measure $(\text { COPM })^{32}$; Australian Therapy Outcome Measure (AusTOMs) ${ }^{33}$, and the Activity Participation Outcome Measure (APOM) ${ }^{34}$. Although these outcome measures have been implemented in monitoring the change in activity participation, it should be acknowledged that the majority were developed in high income countries, as such their usability in a resource-constrained country like South Africa remains unknown.

The literature highlights cost of outcome measures, limited accessibility to context-appropriate measures and time-consuming nature of ROM as some of the leading barriers affecting the implementation of ROM in clinical practice ${ }^{35,36,37}$. While these factors are highlighted as main barriers to ROM, it is important to consider other factors that may influence ROM in clinical practice. In South Africa, the mental health policies emphasize the need to implement evidencebased strategies into practice, however, there are no expectations to produce evidence for practice particularly in government settings ${ }^{21}$. This highlights that while policy outlines the need to demonstrate evidence for interventions through ROM, the implementation of such strategies in clinical practice is viewed as optional. The Rural Mental Health Campaign ${ }^{24}$ describes the state of mental health services in rural areas as dehumanizing. Poor budget allocation and staff shortages have been outlined as the main factors affecting the quality of mental health services within the rural areas ${ }^{22}$. It is undeniable that the mental health services in South Africa fails to meet the needs of the MHCUs as evident with the revolving door phenomena currently crippling the mental health services in the country ${ }^{38}$. Given the current state of mental health services in rural South Africa where quality of care remains questionable, it is critical that emphasis is placed on evidence-based strategies such as ROM to ensure good quality clinical outcomes in mental health ${ }^{26}$.

A deinstitutionalized mental health system, where community care for the MHCUs is strongly advocated, calls for families to become primary caregivers for $\mathrm{MHCUs}^{22}$. With the limited resources and poor quality mental health services reported in rural South Africa, it is important that occupational therapists acknowledge the unique role played by families in the care and management of $\mathrm{MHCUs}^{22,24}$. Families often assume responsibility for the health and well-being of those with mental disorders following discharge from the hospital. They structure daily routines, monitoring the mental state, identify the early signs of illness relapse and deterioration, 
helping the patient to access services, supervising treatment and providing emotional support to the $\mathrm{MHCU}^{39,40,41}$. Through fulfilling these responsibilities, families provide an opportunity for activity participation of MHCUs at the community level. Fulfilling this role is a mammoth task for most families as they are mostly untrained and ill-prepared ${ }^{39}$. Occupational therapists have the knowledge and skills to develop collaborative care models for families to deal with everyday tasks and to ensure that the MHCU can enjoy quality of life despite suffering from a mental disorder. It is, therefore, the authors' opinion that successful implementation of ROM aimed at monitoring outcomes beyond hospitalization requires occupational therapists to form collaborations with the families to help structure routines that will facilitates activity participation in the community.

Literature highlights benefits to ROM and that its implementation within occupational therapy has been a huge task. Although previous research outlines the facilitators and barriers, the evidence to support the implementation of ROM within mental health clinical practice is not well documented. The study aimed to bridge this gap by producing evidence of the changes in activity participation in MHCUs attending an occupational programme through implementing ROM in an acute rural mental health unit in South Africa. This article reports on the amount of change in activity participation as well as the recovery patterns during and after hospitalisation.

\section{METHODOLOGY}

\section{Research design}

A descriptive, longitudinal design with quantitative, quasi-experimental features was employed ${ }^{42}$. Elements of ROM, such as collecting routine data in the clinical setting assessments were conducted in real-life (no variables were manipulated or controlled) with several measurements conducted from baseline to final assessments and data collection focused on one group, pre/post-test design ${ }^{43}$. The quantitative features of the research were investigated by measuring the change in activity participation before and after intervention at different intervention stages. The APOM was administered to determine the level of participation before, during and after intervention. As a quasi- experimental design, the participants became their own controls as this study excluded a randomised sample with an experimental and control group. With no randomised sample, the authors did not claim any cause-and-effect relationship but rather described the changes as measured by the APOM.

\section{Research site}

The study took place in the acute mental health unit of Tintswalo Hospital situated in Mpumalanga Province in the Bushbuckridge area. Tintswalo Hospital is a district hospital with bed capacity of 450 and caters for 16 community clinics. The hospital has the largest mental health unit in the province, with a 54-bed capacity. The bed allocation is estimated at 34 male beds, 10 female beds and eight beds for forensic MHCUs. This unit caters for a variety of patients, ranging from acute to chronic and forensic cases. Due to limited mental health services in the province, the unit caters for a large catchment area, expanded by the introduction of forensic services since 2014 . The average number of inpatients catered for per month is 45 , with admissions amongst males higher than females. At the time of this study a blanket referral was used where each patient admitted to the unit was required to attend occupational therapy and at times referrals were made during the ward rounds. Due to an increase in number of patients the length of stay varied according to the availability of beds and severity of conditions, this was between one to three weeks for acute patients, three to 12 months for long-term and forensic patients. Once discharged into the community MHCUs follow up at the hospital or nearby clinic depending on the area in which they reside. Follow-up of MHCUs is a challenge at this hospital, due to the large catchment area. Often MHCUs are discharged to families outside the province or to the neighbouring country and users then fail to honour follow-up appointments.

\section{Sample method and selection}

Sixty-four MHCUs who were admitted at Tintswalo hospital during data collection participated in this study. A non-probability sampling method was used to select participants. The Raosoft sample size calculator for effect size was used to calculate the sample size ${ }^{44}$. During the time of this study an estimated number of $45 \mathrm{MHCUs}$ were admitted to Tintswalo Hospital per month. To calculate the appropriate sample size for this study the margins of the error were set at $5 \%$ as required for larger groups, with the confidence interval at $95 \%{ }^{44}$. Based on these calculations a minimum sample of 16 was considered adequate to ensure generalisation of the results to the population at Tintswalo Hospital. However, a principle of ROM is to measure the total population, thus using total population sampling. When the total population needs to be included, other factors come into play such as availability of staff to assess all MHCUs, method of assessment and whether the ROM is done as a research project. In the latter case, informed consent is needed which influenced the total population size since only those MHCUs who signed consent, may be included.

No adjustments were made to the occupational therapy programme to align to the focus of this research, as such real-life information was collected without manipulation of the variables. No exclusions were made based on diagnosis, but the common diagnostic groups included schizophrenia, substance use disorder and mood disorder during data collection. Forensic MHCUs were excluded due to the nature and ethical complexity of their cases.

\section{Measurement tool}

The APOM was used for this study. To administer the APOM the researcher attended a one-day training course prior to commencing with the research as required by the developer of the APOM. The APOM was developed and validated by Casteleijn ${ }^{27,34}$ to address the outcome measurement gap for occupational therapy services in mental health within the South African context. The reliability and validity of APOM was conducted in the South African population where a high scale- content validity of 0.93 was established ${ }^{34}$ The responsiveness and sensitivity has been established for people with mental disorders $\mathrm{s}^{21,45,46}$ and those with traumatic brain injury ${ }^{47}$. The APOM is based on the Vona du Toit Model of Creative Ability (VdTMoCA), which consists of nine levels of motivation which corresponds to the action levels ${ }^{15}$ although only the first six levels are incorporated into the APOM. This tool has eight domains, which consists of 53 items to be scored as per the level of creative ability of the client. The domains of APOM includes Process skills, Communication and Interaction skills, Life skills, Role performance, Balanced lifestyle, Motivation, Self-esteem and Affect. Each item on the APOM is described in the level of creative ability and the therapist is required to score the client based on the description they identify to best represent their client and that will determine the level of the client. Once the level is determined, a clinical reasoning process follows where the therapist needs to make a decision about the phase within the level that is therapist-directed, patient-directed or transitional phase. Table I (p66.) presents the 
Table I: The scale of the APOM.

\begin{tabular}{|c|c|c|c|c|c|c|c|c|}
\hline \multicolumn{3}{|c|}{ Level I - Tone } & \multicolumn{3}{|c|}{ Level 2 - Self-differentiation } & \multicolumn{3}{|c|}{ Level 3 - Self-presentation } \\
\hline $\begin{array}{l}\text { Therapist- } \\
\text { directed }\end{array}$ & Patient-directed & Transitional & $\begin{array}{l}\text { Therapist- } \\
\text { directed }\end{array}$ & $\begin{array}{l}\text { Patient- } \\
\text { directed }\end{array}$ & Transitional & $\begin{array}{l}\text { Therapist- } \\
\text { directed }\end{array}$ & $\begin{array}{l}\text { Patient- } \\
\text { directed }\end{array}$ & Transitional \\
\hline 1 & 2 & 3 & 4 & 5 & 6 & 7 & 8 & 9 \\
\hline \multicolumn{3}{|c|}{ Level 4 - Passive participation } & \multicolumn{3}{|c|}{ Level 5 - Imitative participation } & \multicolumn{3}{|c|}{ Level 6 - Active participation } \\
\hline $\begin{array}{l}\text { Therapist- } \\
\text { directed }\end{array}$ & Patient-directed & Transitional & $\begin{array}{l}\text { Therapist- } \\
\text { directed }\end{array}$ & $\begin{array}{l}\text { Patient- } \\
\text { directed }\end{array}$ & Transitional & $\begin{array}{l}\text { Therapist- } \\
\text { directed }\end{array}$ & $\begin{array}{l}\text { Patient- } \\
\text { directed }\end{array}$ & Transitional \\
\hline 10 & 11 & 12 & 13 & 14 & 15 & 16 & 17 & 18 \\
\hline
\end{tabular}

Table II: The Occupational therapy programme at Tintswalo Hospital.

\begin{tabular}{|c|c|c|c|c|}
\hline Monday & Tuesday & Wednesday & Thursday & Friday \\
\hline 08:00 - 09:00 & $08: 00-08: 30$ & $08: 00-09: 00$ & $08: 00-08: 30$ & $08: 00-08: 30$ \\
\hline Rehab meeting & Admin & Psychiatry Meeting & $\begin{array}{l}\text { OT staff Meeting } \\
\text { (rehab) }\end{array}$ & Rixile Health Education \\
\hline 09:00 - 09:30 & $09: 30-13: 00$ & 09:00 - 10:00 & 09:00 - 10:00 & 09:00 - 10:00 \\
\hline OT staff Meeting (rehab) & \multirow[t]{3}{*}{ Ward rounds } & Supervision meeting & $\begin{array}{l}\text { Screening and green } \\
\text { files }\end{array}$ & Admin \\
\hline $10: 00-13: 00$ & & $11: 00-12: 00$ & $11: 00-12: 00$ & $10: 30-12: 00$ \\
\hline Ward rounds & & $\begin{array}{l}\text { Self-care (ADL)* } \\
\text { Gardening*** } \\
\text { Car wash** } \\
\text { Tuck shop** }\end{array}$ & Insight group**** & $\begin{array}{l}\text { Life skills**** } \\
\text { Gardening*** } \\
\text { Car wash*** }\end{array}$ \\
\hline $\mathrm{L}$ & $U$ & $\mathrm{~N}$ & $\mathrm{C}$ & $\mathrm{H}$ \\
\hline $14: 00-15: 00$ & $14: 00-16: 00$ & $14: 00-15: 00$ & $14: 00-16: 00$ & $14: 00-15: 15$ \\
\hline Screening and green files & \multirow[t]{3}{*}{$\begin{array}{l}\text { Active Recreation Group**** } \\
\text { Gardening** } \\
\text { Car wash** }\end{array}$} & $\begin{array}{l}\text { Activity group/ Stimulation } \\
\text { group* } \\
\text { Gardening** } \\
\text { Car wash** } \\
\text { Tuck shop** }\end{array}$ & \multirow[t]{3}{*}{$\begin{array}{l}\text { Activity group*** } \\
\text { Gardening** } \\
\text { Car wash** }\end{array}$} & $\begin{array}{l}\text { Social Skills **** } \\
\text { (unstructured) }\end{array}$ \\
\hline $15: 15-16: 00$ & & $15: 15-16: 00$ & & $15: 30-16: 00$ \\
\hline Admin & & Admin & & Exercise group ${ }^{* * * *}$ \\
\hline
\end{tabular}

*Level I interventions; **Level 2 interventions; ***combined levels (activities adapted for needs of MHCUs).

scale representing the scores used to capture the level as well as the phase of activity participation. The items scores range from I to I8, with I being the lowest activity participation and 18 the highest. Each score on the scale represents a specific level as well as the specific phase within the level for example the score of I to 3 represents the level of Tone while I represents the therapistdirected phase, 2 the patient-directed phase and 3 the transitional phase. The APOM was used to capture five sets of data of the MHCUs gathered through intermittent assessments. Details of the assessment process are presented in the data collection procedure

\section{Data collection procedures:}

The ROM was implemented at Tintswalo hospital and data was collected over six months. The baseline, interim and final assessments were conducted. The APOM was administered on $64 \mathrm{MH}$ CUs attending the occupational therapy programme at Tintswalo hospital. The occupational therapy programme was developed by the occupational therapists who worked in the mental health unit at Tintswalo during the time of this study. The focus of the programme aimed to prepare MHCUs for participation within their home contexts following discharge from the hospital. The intervention was delivered mainly through therapeutic groups, which included self-care (ADL), social/recreational, exercise, life skills, work and insight groups that were structured to cater for MHCUs at different levels of creative ability. In addition to these groups, there were three income-generating projects, which consisted of gardening, tuck shop and care wash. MHCUs were allocated to the appropriate groups based on their level of creative ability. Those who were at lower levels of creative ability such as incidentally constructive level (Level I) were engaged in less structured groups that facilitated reality orientation and exploration of skills. The groups included basic social skills groups such as recreational groups, exercise groups and basic gardening activities such as watering and clearing of weeds. The life skills groups and projects were mainly directed towards MHCUs on the pre-discharge levels, which at Tintswalo hospital ranged between constructive explorative and experimental level of creative ability (Level 2). The therapeutic groups were scheduled to take place during the week starting from Tuesday to Friday. Table II (above) presents the occupational therapy programme (the shaded areas indicate the intervention part of the programme).

All eight domains were measured, and five sets of data were captured in the form of intermittent assessment and recording the findings on the APOM. The researcher along with two other occupational therapists who worked at the mental health unit conducted 
Table III: Assessment methods used for data collection.

\begin{tabular}{|c|c|c|}
\hline $\begin{array}{l}\text { Baseline assessment } \\
\text { (Assessment I) }\end{array}$ & $\begin{array}{l}\text { Intermediate and discharge Assessment } \\
\text { (Assessment } 2 \text { and } 3 \text { ) }\end{array}$ & $\begin{array}{l}\text { Follow-up and Final Assessment } \\
\text { (Assessment } 4 \text { and 5) }\end{array}$ \\
\hline \multicolumn{3}{|c|}{ Interview(questioning), observations and collateral information } \\
\hline \multicolumn{3}{|l|}{ Activities used } \\
\hline $\begin{array}{l}\text { Soccer } \\
\text { Duster hockey } \\
\text { Card making } \\
\text { Insight games } \\
\text { Board games } \\
\text { (Morabaraba, draughts) }\end{array}$ & $\begin{array}{l}\text { Gardening } \\
\text { Baking } \\
\text { Card making } \\
\text { Insight games } \\
\text { Puzzles } \\
\text { Lego } \\
\text { Home maintenance tasks (e.g. cleaning) }\end{array}$ & $\begin{array}{l}\text { Key ring } \\
\text { Envelope making } \\
\text { Word search } \\
\text { Maze } \\
\text { Puzzles } \\
\text { Beading (bracelets and necklaces) } \\
\text { Home programmes (cleaning, cooking gardening) }\end{array}$ \\
\hline
\end{tabular}

Table IV. Demographics of the sample $(n=64)$.

\begin{tabular}{|c|c|c|c|c|c|}
\hline Age in Years & Category & Male $(n=48)$ & $\%$ & Female $(n=16)$ & $\%$ \\
\hline$<20$ & I & 2 & 3.13 & 1 & 1.56 \\
\hline $20-29$ & 2 & 27 & 42.19 & 7 & 10.94 \\
\hline $30-39$ & 3 & 12 & 18.75 & 5 & 7.81 \\
\hline $40-49$ & 4 & 6 & 9.38 & 2 & 3.13 \\
\hline $50-59$ & 5 & I & 1.56 & I & 1.56 \\
\hline \multicolumn{6}{|l|}{ Diagnoses } \\
\hline Schizophrenia & I & 19 & 29.69 & 4 & 6.25 \\
\hline Bipolar Mood Disorder & 2 & 6 & 9.38 & 7 & 10.94 \\
\hline Substance use disorder & 3 & 20 & 31.25 & 0 & 0 \\
\hline Other (epilepsy, acute psychosis) & 4 & 3 & 4.69 & 5 & 7.81 \\
\hline
\end{tabular}

assessments. Intervention followed through facilitating activity participation in the occupational therapy programme as per routine. The researcher was the only person responsible for the scoring of the APOM items for the individual mental healthcare users. The two therapists then assessed and provided interventions as usual. For MHCUs in the ward assessments took place in the group context and for outpatient follow-up assessments were done individually. Table III (above) presents a summary of activities used for the assessment of clients throughout the data collection process.

For baseline assessments an APOM was administered in the first week of attending the occupational therapy programme. During this stage, participants were involved in five group sessions that were facilitated throughout the week and included engagement in self-care, social skills, work-related tasks, insight, and recreational groups. The groups provided opportunity for the observation of participants' activity participation in different contexts, which was then used to establish the baseline level of activity participation.

The interim assessment varied based on the length of stay ranging between two to three weeks of hospitalisation. Therefore, for other participants interim APOM assessments were conducted after two weeks, while for others after three weeks of hospitalisation. At this stage, the focus of assessments was placed on handling of tools, materials and situations, behaviour and interaction with others, amount of assistance needed and nature of engagement in all activities.

For the third assessments, an APOM was administered when participants were discharged from the hospital. This assessment determined the level of change in activity participation from baseline and marked as point of reference for the researcher to evaluate activity participation within the participant's home context during follow up phases. Two follow-up sessions were scheduled i.e. follow-up one and final assessment. The follow-up sessions took place at the occupational therapy department at Tintswalo hospital and were carried out when MHCUs returned for the doctor's follow-up visits. The first follow-up APOM assessment was administered between two to four weeks post discharge depending on the return dates given by the doctor during discharge. The final assessment is the fifth and last assessment in the study. For participants who were required to return to the doctor at Tintswalo hospital, the final assessment date was given corresponding to the next doctor's follow-up visit. However, for those who were referred to the local clinics for further management, dates were agreed upon based on their availability to return to the hospital. The APOM was administered after one month after the follow-up assessment. For both follow-up assessments participants were involved in a 45-minute individual session and collateral information was obtained from the family to complete the APOM. Assessments entailed involving MHCUs in activities such as making a keyring, word search tasks, beading activities and cooking activities. Additionally, home programmes given to MHCUs during discharge were reviewed and participation was assessed based on input from the family members who accompanied the MHCUs. Data for the five assessments was captured first on the hard copy then transferred onto an Excel version of APOM for further analysis.

\section{Data analysis}

Quantitative review of data calls for the condensation of results from several data sheets into a single data sheet. Descriptive statistics were used for the description of demographic data. Pooled effect sizes, a name which is given to a family of indices that mea- 


\section{Average APOM score per domain at each data collection point}

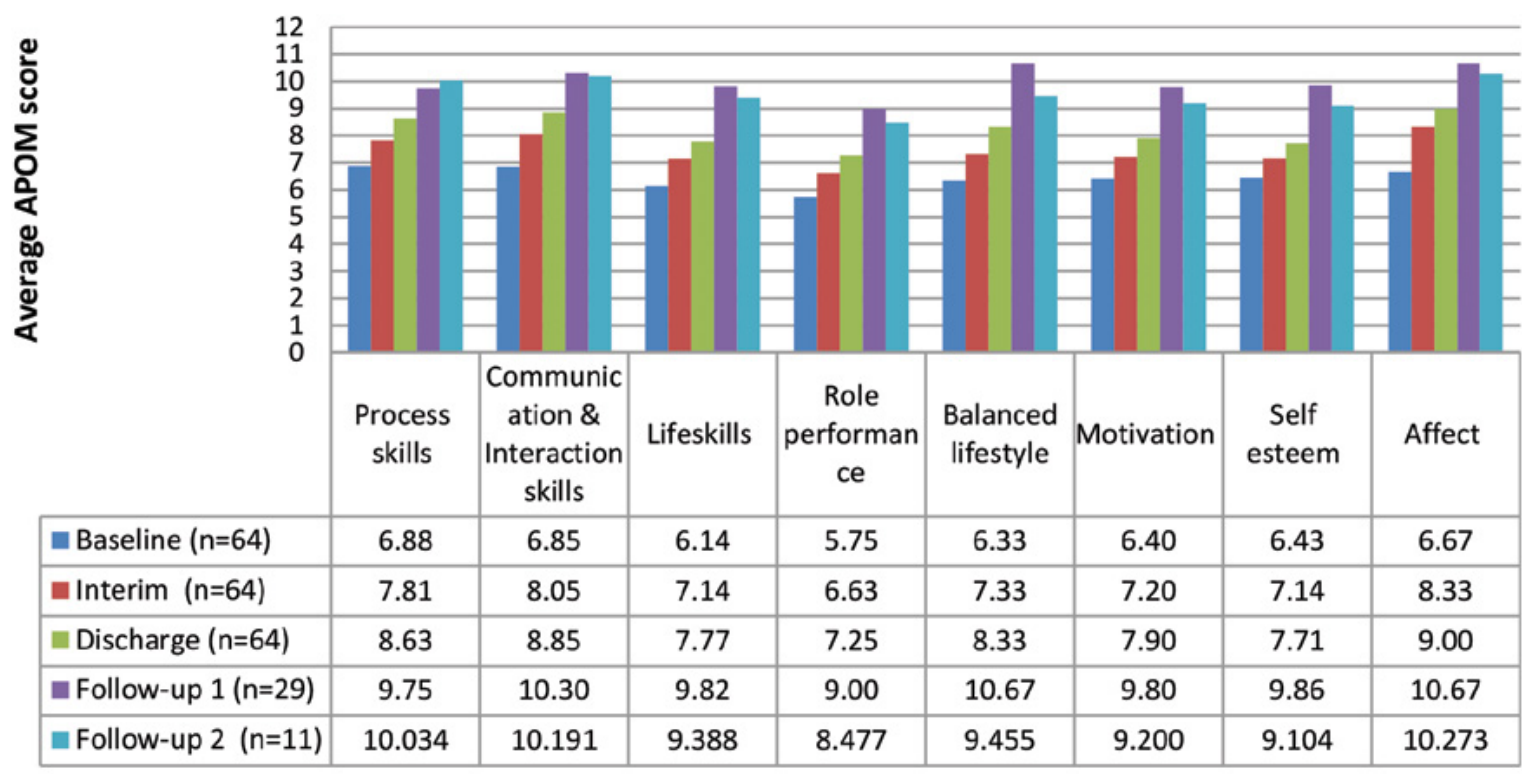

Figure I:

Average APOM score per domain at each data collection point. sure the magnitude of treatment effect ${ }^{48}$, were used to describe trends in changes in activity participation. Internal responsiveness of the study was established through calculation of the effect size of the participants' activity participation. Cohen's $d$ and Cohen's $r$ effect size statistics were used to determine standardized differences between assessments in this study. Cohen's $d$ was done by calculating the difference between the mean baseline scores and follow-up scores, which was then divided by the standard deviation of baseline scores ${ }^{49}$. To interpret the results using the Cohen's $d$ the suggested $d$ values were used as guidelines whereby value of 0.20 is considered small, 0.5 medium and 0.80 large effect sizes ${ }^{50}$. Cohen's $r$ was determined from a table by converting the $d$ value to a correlation ${ }^{50}$. The suggested value for Cohen's $r$ is 0.10 (small effect), 0.24 (medium effect) and 0.37 large effect) ${ }^{50}$. Statistical significance of the change was determined with the Wilcoxon matched pairs test with the $p$-value set at $<0.05$.

\section{RESULTS}

\section{Demographics}

Table IV (p67) documents the demographics of the sample $(n=$ 64). The demographics of the study reflected that one third of the sample was females and two thirds males, with most of the participants (42.19\%) between the ages of $20-29$ followed by those with an age range of $30-39(18.75 \%)$. Amongst common groups of diagnoses investigated, majority of the participants were those diagnosed with substance use disorders (31.25\%) followed by those with schizophrenia (29.69\%).

\section{Change in levels of activity participation from baseline to final assessments}

Figure I documents the changes in activity participation from baseline to final assessment. The results indicate improvement in levels of activity participation in all domains, from baseline to discharge. Important to note is the changes in sample size. There were no changes in the sample size for the hospitalisation period (baseline, interim and discharge), but loss to follow-up from discharge to the first follow-up was 35 (54, 68\%) MHCUs. Loss to follow-up between the two follow-up points was 18 (62\%) MHCUs.

Figure 2 (p69) documents Cohen's $d$ and Cohen's $r$ effect sizes during hospitalisation, the large values $(>0.80)$ in the effect sizes demonstrated a substantial change in activity participation $(n=64)$. The change was highly statistically significant with a $p$ value of 0.000 for all the domains for the hospitalisation phase.

Figure 3 (p69) documents Cohen's $d$ and Cohen's r effect sizes between discharge and follow-up 2 assessment periods, these results demonstrate low effect sizes $(<0.20)$ which demonstrates a substantial decline in other activity participation domains including motivation, while other domains including affect and life skills were maintained. The $p$-values were statistically insignificant for all the domains and ranged between 0.878 and 0.091 .

\section{DISCUSSION}

Majority of the participants were males between the ages of 20-29. This is not surprising considering that Tintswalo hospital mental health unit has a higher bed allocation for males than females. Substance use disorder was identified as a leading diagnosis followed by schizophrenia. In Mpumalanga province, a high rate of substance abuse is reported amongst males between the ages of $15-39^{51}$. According to McLellan ${ }^{52}$ substance abuse is identified as a precipitating factor to substance related mental disorders. The prevalence of substance use disorders in the country is uncertain and ranges between $5.8 \%$ as reported in 2013 in the $\mathrm{NMHF}^{23}$ to $15 \%$ in 2017 as reported by Recovery Direct ${ }^{53}$. Given the fact that substance abuse is identified as a major problem affecting rural black communities within South Africa, it is expected that majority of the participants within the population studied presents with substance use disorders ${ }^{54}$.

The fact that most of the participants were between ages 20-29 raises a serious concern. This stage of development is marked as entry point into worker roles with the requirement of forming and maintaining a productive and self-satisfying career ${ }^{55}$. Unemployment rate amongst patients with severe mental disorders like schizophrenia and substance use disorders is estimated between $80 \%$ to $90 \%{ }^{56}$. According to Bonder et al. ${ }^{57}$, Schizophrenic diagnosis and psychotic symptoms serve as predictors of low employability amongst this 


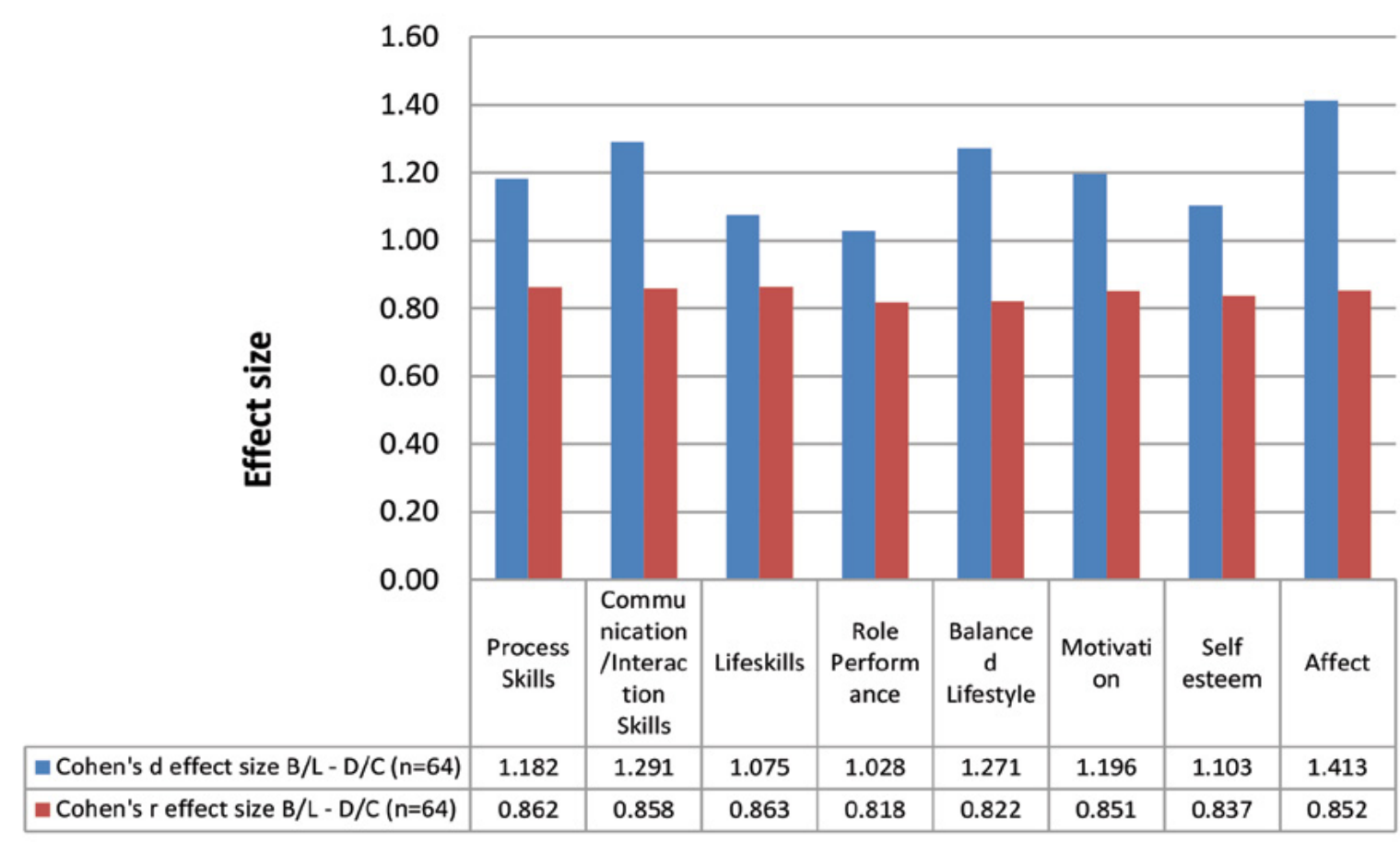

Figure 2: Cohen's

d and Cohen's

$r$ effect sizes

between baseline

and discharge

assessment periods

$(n=64)$.

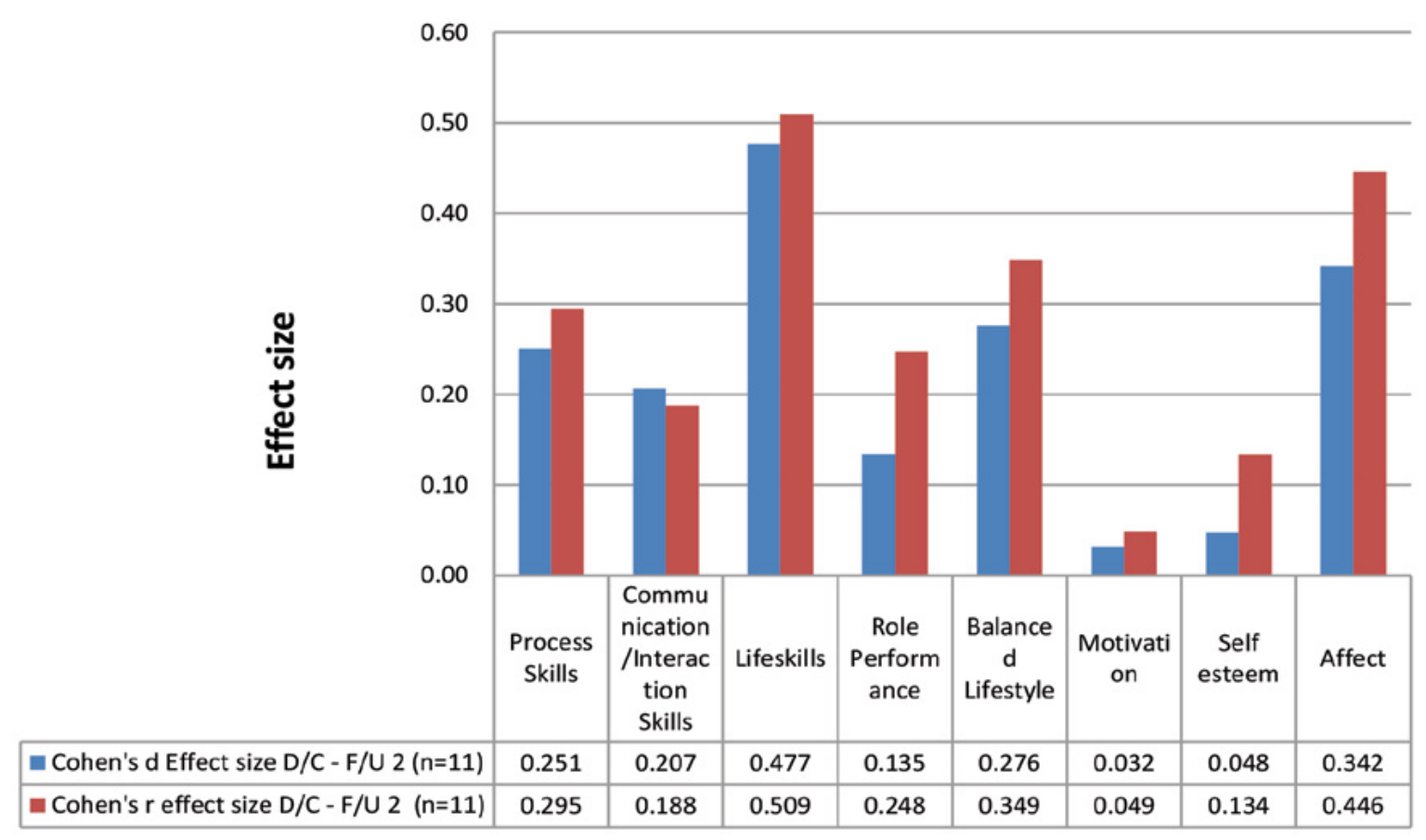

Figure 3:

Cohen's $d$ and

Cohen's $r$ effect

sizes between

discharge and

follow-up 2

assessment

periods.

population. This therefore indicates that majority of the participants are highly unlikely to secure employment in the open-labour market ${ }^{56}$. The issues of unemployment amongst those with MHCUs in rural areas are further compounded by the high rates of unemployment within South Africa. Mpumalanga province is mostly rural and identified to have second highest rate of unemployment of $43 \%$ within the entire country ${ }^{58}$. To ensure that the MHCUs in this context remain productive occupational therapy programmes should incorporate prevocational skill training to ensure productivity as required for this age group.

\section{Description of levels of activity participation before and after occupational therapy intervention}

The APOM mean scores indicated substantial improvement across all domains during hospitalization (baseline to discharge). In contrast to this, the mean scores from discharge to final follow-up assess- ment demonstrated a decline in activity participation. The APOM mean scores ranged from patient-directed incidentally constructive action (self-differentiation) level of creative ability at baseline (5.75) to transitional constructive explorative action (self- presentation) level of creative ability at discharge phase (9.00). The scores for Role performance were the lowest and for Affect, the highest. These results represent significant change during the hospitalization phase.

The established incidentally constructive action (self-differentiation) level of creative ability at baseline represents the level patients regress to during their acute stage of illness amongst the population at Tintswalo hospital. Studies conducted in the $\mathrm{UK}^{46}$ and at Sterkfontein hospital ${ }^{45}$ in South Africa that used the APOM, demonstrated overall level of constructive explorative action (self-presentation) at baseline assessments. These studies were done in different contexts, but it was still useful to compare the baselines of the studies that used the APOM to track change in MHCUs as this has not been done previously. From 
the findings of our study, it is evident the baseline APOM scores at Tintswalo hospital are slightly lower than other settings. According to Casteleijn and Holsten ${ }^{59}$, an incidentally constructive action level of creative ability is characterized by disorganized thinking, monosyllabic communication, aggressive and bizarre or uncontrollable behavior. Patients on this level are likely to be seen in chronic mental health institutions where structured rehabilitation programmes are provided with 24-hour care. Tintswalo Hospital, as an acute unit, varies from other settings, catering for a variety of patients of whom majority are chronic and often take longer to recover, therefore it is not surprising that this level was established at baseline.

While the study yielded positive results during hospitalisation, it is very important to note the negative implications that comes with the discharge of patients on the constructive explorative action level of creative ability. According to VdTMoCA patients on this level require assistance to function within their communities. Although they express desire for independent living patients on this level require support to gain and maintain productive lives ${ }^{59}$. They need supervision to engage in activities of daily living such as caring for self, personal belongings, and their immediate surroundings. They are unable to sustain effort when performing tasks which results in decreased productivity and social behavior is often inconsistent as they often disregard the feelings of others ${ }^{59}$. Van der Reyden et al. ${ }^{15}$ point out that a MHCUs achieve skills for independent living once they reach the experimental (passive participation) level of creative ability. Therefore, this implies that the MHCUs at Tintswalo hospital were discharged prior to attaining the necessary skills required for independent living within their community. Various factors could be attributed to the discharge of MHCUs at this level, it is the authors' perspective that the care of MHCUs at Tintswalo hospital is governed by the medical model where the focus is on alleviating the distressing psychotic symptoms than function. Based on the observation of the author, MHCUs were discharged once their psychotic symptoms settled without consideration of the level of skills for independent community living. This has serious implications for the families as primary caregivers for this MHCUs. For MHCUs who are unable to function independently their families are expected to manage behavior, structure routines and monitor treatment of the $\mathrm{MHCU}^{39,41}$. According to $\mathrm{Chadda}^{39}$, the role as caregiver is a mammoth task for which family members are mostly untrained and unprepared. The responsibility of supporting the MHCUs is a traumatic and burdensome experience which can lead to significant levels of distress ${ }^{60}$. Given the fact that MHCUs in this study were discharged before they could attain the necessary skills for independent living it could be assumed that a decline in activity participation noted during the follow-up stages of this study could be related to the distress experienced by the families.

One of the values and principles outlined by the $\mathrm{NMHF}^{23}$ is the provision of maximum support to the families and caregivers of those with mental illness, to broaden the network of support and care. To achieve this the South African health system needs to develop and strengthen the community mental health services that will offer support to the MHCUs and their families in rural settings. Mapanga et al. highlight three top strategies in their evidence map for strengthening provision of mental health services within PHC level to support community reintegration of MHCUs. The top strategy was empowerment of families, carers, and patients with integration of care or collaborative interventions second and thirdly, the use of e-health services. The inclusion of families, carers and patients in the treatment process, self-help interventions, support groups, psychoeducation, counselling and addressing caregiver burden has been associated with good outcomes for the MHCUs ${ }^{61}$. Therefore, it is important that the focus of mental health services shift to incorporating interventions for families and caregivers of people with mental illness particularly in rural areas where mental health services are almost non-existent. This can be done through psychoeducation, psychosocial rehabilitation, and provision of home programmes to assist families in structuring the activities of the MHCUs. The recovery and well-being of the MHCU is linked to that of their caregiver ${ }^{61}$, it is crucial that occupational programmes in mental health incorporate strategies focused specifically on meeting the needs of the families. Successful implementation these strategies will not only improve patient outcomes but also alleviate pressure from the burdened mental health system.

\section{Recovery patterns in activity participation}

Effect sizes demonstrated improvement with large values on both Cohen's $d$ and Cohen's $r$ statistics during hospitalization and showed clinical significance of the study. These results indicate a positive change in activity participation across all domains. The results from discharge to final assessment yielded slight change in activity participation, which was accompanied by a decline in activity participation in other domains as evident in the values of APOM scores and effect sizes (see Figure 3). From these results, it is evident the decline started after the first follow-up, thus four to six weeks after discharge. Currently, there is no literature to justify the deterioration in APOM scores following discharge from the hospital. However, in this study, deterioration was noted, and the following reasons were identified: by the time, they came back for a follow up other participants had relapsed due to poor compliance to medication caused by lack of supervision, while others continued to use substances while taking medication. This is attributed to the fact that most patients were discharged without insight to their conditions. Some MHCUs lacked structure to engage in activities of daily living within their home environment as family members viewed these MHCUs as incapable of carrying out such tasks. Although attempts were made to help provide the structure for activity participation in the home environment through home programmes, however, this was unsuccessful as some of the programmes were not monitored by the family. The lack of willingness and involvement of the family in the management of these MHCUs could be related to the fact that they were not supported to develop the necessary skills that are required to provide care to the $\mathrm{MHCU}$ within their care. The home programmes focused solely on the activities that the families had to structure to facilitate activity participation instead of ways in which the family could cope with some of the distressing behaviour exhibited by the MHCUs.

Despite a substantial decrease in sample size during the follow-up stages the results of this study provide evidence to suggest that while occupational therapy programmes succeed during hospitalization these changes may not be sustainable after discharge. In this study, the changes made during this hospitalization were sustained after one month but not after three months. The recovery principle outlined in the $\mathrm{NMHF}^{23}$ emphasises the need for services and delivery to aim at building the MHCUs' ability to return to, sustain and participate in satisfying roles of their choice in their community. The decrease in sample size and slight decline in some of the APOM domains noted in the final follow up stages of this study raises a concern regarding the mental health service delivery in rural South Arica. This could highlight failure of these services in supporting MHCUs to re-integrate within their communities following discharge. This can be highlighted as a contributing factor towards the revolving door phenomenon that is 
crippling the services in the country.

\section{LIMITATIONS OF THE STUDY}

As a quasi-experimental design, this study only consisted of onegroup from the same hospital with no randomisation of the sampling which limits the generalizability of the results. The significant decrease in the sample size noted in especially the final assessments (three months after discharge) of this study narrowed the results; therefore, no significant conclusions could be drawn for patterns of change after discharge.

\section{CONCLUSION}

This was the first study that establishes effect sizes in activity participation in MHCUs in a rural setting and can therefore be used as a benchmark for acute settings such as Tintswalo hospital. The value of measuring the change in activity participation through implementing ROM in real life clinical situations was apparent and valuable practical conclusions were reached even without a control group. The effect of the occupational therapy programme on the activity participation of MHCUs during hospitalisation phase was established. Areas needing improvement in the programme were identified, which included facilitation of activity participation to improve role performance amongst the population at Tintswalo. The results noted in the follow-up stage provided an opportunity for exploring ways in which these services can be facilitated for continued activity participation within the environmental contexts of the MHCUs. One way in which this could be implemented is through development of home programmes which will focus on the needs of both the family and the MHCU to help structure the activities for MHCUs in their home environment. There is an urgent need for the focus of mental health services to move towards development and strengthening of community-based rehabilitation to facilitate successful reintegration to the community and offer support to the families of the MHCUs particularly in rural areas where services are limited.

\section{ACKNOWLEDGEMENTS}

Tintswalo MHCUs for participating in the study. Denise Franzsen for her assistance with the statistical analysis. As a Consortium for Advanced Research Training in Africa (CARTA) scholar we wish to acknowledge CARTA for an exceptional training programme that has enhanced my academic writing skills.

\section{REFERENCES}

I. Meffert SM, Neylan TC, Chambers DA, Verdeli H. Novel implementation research designs for scaling up global mental health care: overcoming translational challenges to address the world's leading cause of disability. International Journal of Mental Health Systems. 2016;10(I):19. https://dx.doi.org/10.1 I86/s 13033-016-0049-7.

2. Vigo $D$, Thornicroft $G$, Atun R. Estimating the true global burden of mental illness. The Lancet Psychiatry. 2016;3(2): I7I-8

https://dx.doi.org/https://doi.org/10.1016/S2215-0366(I5)00505-2.

3. South African National Department of Health. Government Notices no 40955 - National Health Insurance Policy Pretoria; 2017. Retrieved from https://www.gov.za/sites/default/files/ gcis_document/201707/40955gon627.pdf

4. Herman AA, Stein DJ, Seedat S, Heeringa SG, Moomal H, Williams DR. The South African Stress and Health (SASH) study: I2-month and lifetime prevalence of common mental disorders. South African Medical Journal. 2009;99(5). https://www.ajol.info/index.php/samj/ article/view/50764
5. Rudnick A. What is a Psychiatric Disability? Health Care Analysis. 2014;22(2): I05- 13 https://dx.doi.org/10. 1007/s I0728-0I 2-0235-y

6. American Occupational Therapy Association. Occupational therapy's distinct value: Mental health promotion, prevention, and intervention across the lifespan 2016. [Available from: https://www. aota.org/-/media/Corporate/Files/Practice/MentalHealth/DistinctValue-Mental-Health.pdf]

7. Bjørkedal STB, Bejerholm U, Eplov LF, Møller T. Meaningful Activities and Recovery (MA\&R): the effect of a novel rehabilitation intervention among persons with psychiatric disabilities on activity engagement—study protocol for a randomized controlled trial. Trials. 2020;2 I (I):789 https://dx.doi.org/I 0. I I86/s / 3063-020-04722-3

8. Baker AE, Procter N, Gibbons T. Dimensions of loss from mental illness. J Soc \& Soc Welfare. 2009;36:25 https://scholarworks.wmich. edu/jssw/vol36/iss $4 / 4$

9. Bejerholm U, Roe D. Personal recovery within positive psychiatry. Nordic Journal of Psychiatry. 2018;72(6):420-30 https://dx.doi.org /10.1080/08039488.2018.14920I5

10. Doroud N, Fossey E, Fortune T. Recovery as an occupational journey: A scoping review exploring the links between occupational engagement and recovery for people with enduring mental health issues. Australian Occupational Therapy Journal. 2015;62(6):378-92 https://dx.doi.org/https://doi.org/10.1 I I I/1440-1630.12238

II. Lannigan EG, Noyes S. Occupational Therapy Interventions for Adults Living With Serious Mental Illness. American Journal of Occupational Therapy. 2019;73(5):73053950 I0pI-p5 https://dx.doi.org//0.5014/ajot.2019.73500।

12. Casteleijn D. The 22nd Vona du Toit Memorial Lecture: Stepping Stones from Input to Outcomes: An Occupational Perspective. South African Journal of Occupational Therapy. 20I3;43:0I-9.

13. Creek J, Lougher L. Occupational therapy and mental health. Philadelphia: Churchill Livinstone Elsevier $201 \mathrm{I}$.

14. American Association of Occupational Therapy. OCCUPATIONAL THERAPY PRACTICE FRAMEWORK: Domain \& Process, 3rd Edition. American Journal of Occupational Therapy. 20I4:SI-S48 https://dx.doi.org/http://dx.doi.org/I0.50I4/ajot.20I4.682006

15. Van der Reyden D, Casteleijn D, Sherwood W, De Witt P. The Vona du Toit Model of Creative Ability: Origins, Constructs, Principles and Application in Occupational Therapy. Pretoria: Vona and Marié du Toit Foundation; 2019.

16. Dijkers MP. Issues in the Conceptualization and Measurement of Participation: An Overview. Archives of Physical Medicine and Rehabilitation. 2010;91(9, Supplement):S5-SI6 https://dx.doi.org/https://doi.org//0.1016/j.apmr.2009.10.036

17. World Health Organisation. International Classification of Functioning, Disability and Health (ICF) 200 I [Available from:

https://www.who.int/standards/classifications/international-classification-of-functioning-disability-and-health].

18. Law M. Participation in the Occupations of Everyday Life. American Journal of Occupational Therapy. 2002;56(6):640-9 https://dx.doi.org// 0.50I4/ajot.56.6.640

19. Bryant W, Cordingley K, Sims K, Dokal-Marandi J, Pritchard H, Stannard $\mathrm{V}$, et al. Collaborative research exploring mental health service user perspectives on acute inpatient occupational therapy. British Journal of Occupational Therapy. 2016;79(10):607-13 https://dx.doi.org/10.1 I77/0308022616650899

20. Foruzandeh N, Parvin N. Occupational therapy for inpatients with chronic schizophrenia: A pilot randomized controlled trial. Japan Journal of Nursing Science. 2013; 10(I):136-4I https://dx.doi.org/https://doi.org/10.111 I/j.17427924.2012.00211.x. 
21. Silaule O. Routine measurement of outcomes for mental health care users attending occupational therapy [MSc Research report]: University of the Witwatersrand; 2016. [Retrieved from http://hdl. handle.net/l0539/23305]

22. Vergunst R. From global-to-local: rural mental health in South Africa. Global Health Action. 2018; I I(I): 1413916

https://dx.doi.org/10.1080/165497/6.2017.1413916

23. South African National Department of Health. National Mental Health Policy Framework and Strategic Plan 2013-2020. 2012. Retrieved from https://www.health-e.org.za/wp-content/uploads/2014/I0/National-Mental-Health-Policy-Framework-andStrategic-Plan-2013-2020.pdf

24. Rudasa. The Rural Mental Health Campaign Report. 2015. Retrieved from https://static.pmg.org.za/I 703 I 5RURALMENTAL.pdf

25. Essock SM, Olfson M, Hogan MF. Current practices for measuring mental health outcomes in the USA: International overview of routine outcome measures in mental health. International Review of Psychiatry. 20I5;27(4):296-305 https://dx.doi.org/10.3109/0954026I.2015.1014314

26. Kilbourne AM, Beck K, Spaeth-Rublee B, Ramanuj P, O'Brien RW, Tomoyasu N, et al. Measuring and improving the quality of mental health care: a global perspective. World Psychiatry. 2018; I (I):30-8 https://dx.doi.org/https://doi.org// 0.1002/wps.20482

27. Casteleijn $\mathrm{D}$. Using measurement principles to confirm the levels of creative ability as described in the Vona du Toit Model of Creative Ability. South African Journal of Occupational Therapy. 20I4;44:149. https://dx.doi.org/https://www.sajot.co.za/index.php/sajot/article/ view/228/126

28. Wong L, Harris M, Cotton S, Edwards J. Routine outcome assessment and feedback for clinicians: A pilot in an early psychosis service. Journal of Mental Health. 2006; I5(3):279-88

https://dx.doi.org//0.1080/096382306007/4285

29. Carlier IV, van Eeden WA. Routine Outcome Monitoring in Mental Health Care and Particularly in Addiction Treatment: EvidenceBased Clinical and Research Recommendations. Journal of Addiction Research \& Therapy. 2017;8:332

https://dx.doi.org/10.4172/2155-6105.1000332

30. Kramer J, Kielhofner G, Lee SW, Ashpole E, Castle L. Utility of the Model of Human Occupation Screening Tool for Detecting Client Change. Occupational Therapy in Mental Health. 2009;25(2): I8I-9I https://dx.doi.org/l0.1080/01642 I 2090285926I

31. Park S, Fisher AG, Velozo CA. Using the Assessment of Motor and Process Skills to Compare Occupational Performance Between Clinic and Home Settings. American Journal of Occupational Therapy. 1994;48(8):697-709 https://dx.doi.org/10.5014/ajot.48.8.697

32. Law M, Baptiste S, McColl M, Opzoomer A, Polatajko H, Pollock N. The Canadian Occupational Performance Measure: An Outcome Measure for Occupational Therapy. Canadian Journal of Occupational Therapy. 1990;57(2):82-7 https://dx.doi. org/10.1 I77/00084I749005700207

33. Unsworth CA. Measuring Outcomes using the Australian Therapy Outcome Measures for Occupational Therapy (AusTOMs-OT): Data Description and Tool Sensitivity. British Journal of Occupational Therapy. 2005;68(8):354-66 https://dx.doi.org/10.1 177/030802260506800804

34. Casteleijn JMF. Development of an outcome measure for occupational therapists in mental health care practice. PhD: University of Pretoria; 2010. [Retrieved from http://upetd.up.ac.za/thesis/available/etd-02 I020I I-143303/]

35. Boswell JF, Kraus DR, Miller SD, Lambert MJ. Implementing routine outcome monitoring in clinical practice: Benefits, challenges, and solutions. Psychotherapy Research. 2015;25(I):6-19.

https://dx.doi.org/https://doi.org//0.1080/I0503307.2013.8I7696.

36. Casteleijn D, Graham M. Domains for occupational therapy outcomes in mental health practices. South African Journal of Occupational Therapy. 2012;42(I):26-34.

37. Trauer T. Outcome measurement in mental health: theory and practice. Cambridge: Cambridge University Press; 2010.

38. South African Human Rights Commision. Annual report 2017. Retrieved from https:/www.sahrc.org.za/home/2I/files/SAHRC\%20 Annual\%20Report\%202017\%20HR.PDF

39. Chadda RK. Caring for the family caregivers of persons with mental illness. Indian J Psychiatry. 2014;56(3):221-7

https://dx.doi.org/10.4103/0019-5545.1406/6

40. Mokgothu MC, Plessis ED, Koen MP. The strengths of families in supporting mentally-ill family members : original research. Curationis. 20I5;38(I): I-8 https://dx.doi.org/doi:10.4102/curationis.v38il.1258.

41. Paruk S, Ramdhial M. Prevalence of caregiver burden, depressive and anxiety symptoms in caregivers of children with psychiatric disorders in Durban, South Africa. South African Journal of Psychiatry. 2018;24(I): I https://dx.doi.org/doi:10.4102/sajpsychiatry.v24i0.1314

42. De Vos AS, Strydom H, Fouché CB, Delport CSL. Research at Grass roots for the Social Sciences and Human Service Professions. 3rd ed. Pretoria: Van Schaick publishers; 2007.

43. Tomlin G, Borgetto B. Research Pyramid: A New Evidence-Based Practice Model for Occupational Therapy. American Journal of Occupational Therapy. 201 I;65(2): 189-96 https://dx.doi.org//0.5014/ajot.201।.000828

44. Raysoft sample size calculator 2004 [Retrieved from: http://www.raosoft.com/samplesize.html]

45. Brooke CA. Selected psychometric properties of the activity participation outcome measure to describe trends in a forensic population of mental health care users: University of the WItwatersrand; 2015. [Retrieved from http://wiredspace.wits.ac.za/handle/I0539//8684]

46. Carter M. Analysis of routine outcome measurement data in mental health occupational therapy. [MSc Research report]: University of Northampton.; 2013

47. Camp AL, Casteleijn D, Thupae D. Responsiveness of the Activity Participation Outcome Measure in adult patients with Traumatic Brain Injury in an acute private neurological rehabilitation setting in South Africa. South African Journal of Occupational Therapy. 2020;50:70-6 https://dx.doi.org/http://dx.doi.org//0.17/59/23103833/2020/vol50no2a9

48. Becker LA. Effect size (ES) 2000 [Available from: https://www.uv.es/ friasnav/EffectSizeBecker.pdf]

49. Husted JA, Cook RJ, Farewell VT, Gladman DD. Methods for assessing responsiveness: a critical review and recommendations. Journal of Clinical Epidemiology. 2000;53(5):459-68 https://dx.doi.org/https://doi.org/10.1016/S0895-4356(99)00206-I

50. Fritz CO, Morris PE, Richler JJ. Effect size estimates: Current use, calculations, and interpretations. Journal of Experimental Psychology: General. 2012;14I(I):2 - 18 https://dx.doi.org/https://doi.org//0.1037/a0024338

5I. Dada S, Harker Burnhams N, J. E, Warren Lucas W, Parry C, Bhana $A$, et al. Monitoring alcohol, tobacco and other drug abuse treatment admissions in South Africa. South African Community Epidemiology Network on Drug Use (SACENDU); 2017. Retrieved from https://www.samrc.ac.za/sites/default/files/attachments/2020-II-I8/SACENDUFullReportPhase47.pdf

52. McLellan AT. Substance Misuse and Substance use Disorders: Why 
do they Matter in Healthcare? Trans Am Clin Climatol Assoc. 2017; 128: I 12-30.

https://dx.doi.org/https://www.ncbi.nlm.nih.gov/pmc/articles/ PMC5525418/

53. Recovery Direct. The Staggering Stats of Addiction in South Africa 2017 [Retrieved from:

https://www.recoverydirect.co.za/staggering-stats-addiction-southafrica/]

54. Oladeinde O, Mabetha D, Twine R, Hove J, Van Der Merwe M, Byass $P$, et al. Building cooperative learning to address alcohol and other drug abuse in Mpumalanga, South Africa: a participatory action research process. Global Health Action. 2020; I3(I): 1726722 https://dx.doi.org/10.1080/165497/6.2020.1726722

55. Halfon N, Forrest CB. The Emerging Theoretical Framework of Life Course Health Development. Handbook of life coure health development. 2018; 19-43. London: Springer Nature.

56. Mueser KT, Deavers F, Penn DL, Cassisi JE. Psychosocial Treatments for Schizophrenia. Annual Review of Clinical Psychology. 20। 3;9(I):465-97 https://dx.doi.org/I0.I I 46/annurev-clinpsy-0502 I 2-I85620

57. Bonder BR, Martin L, Miracle AW. Culture Emergent in Occupation. American Journal of Occupational Therapy. 2004;58(2):159-68 https://dx.doi.org/10.50I4/ajot.58.2.159

58. StatsSA. Quarterly Labour Force Survey In: Statistics South Africa, editor. Pretoria2019

59. Casteleijn D, Holsten E. Creative Ability - its emergence and manifestation. In: Van der Reyden D, Casteleijn D, Sherwood W, De Witt P, editors. The Vona du Toit Model of Creative Ability: Origins, Constructs, Principles and Application in Occupational Therapy Pretoria: Vona and Marié du Toit Foundation; 2019. Pretoria: Vona and Marié du Toit Foundation; 2019.

60. Stomski NJ, Morrison P. Predictors of burden in Australian mental health caregivers: a cross-sectional survey. Journal of Mental Health. 202I;30(4):4II-6

https://dx.doi.org/10.1080/09638237.2019.1581340

6I. Mapanga W, Casteleijn D, Ramiah C, Odendaal W, Metu Z, Robertson $L$, et al. Strategies to strengthen the provision of mental health care at the primary care setting: An Evidence Map. PLOS ONE. 2019; |4(9):e0222162

https://dx.doi.org/10.1371/journal.pone.0222162

\section{Corresponding Author}

* Olindah Silaule

Email: olindah.silaule@wits.ac.za 